\title{
Costos ambientales en el proceso de extracción del aceite de palma. Estudio de un caso
}

Reinosa Pulido, Doris C.*

\section{Resumen}

El objetivo de la presente investigación es establecer los factores que inciden en la conformación de los costos ambientales en el procesamiento de extracción del aceite de palma en la planta procesadora Palmeras San Simón, en el Municipio Colón, Estado Zulia. La investigación se enmarca dentro de la contabilidad financiera, sobre la base de los costos internos. El tipo de investigación es descriptiva, de campo, las técnicas utilizadas fueron la entrevista, la observación directa y el cuestionario. Se obtuvieron los siguientes resultados: 1) La empresa conoce los principales efluentes, impacto ambiental y ha establecido acciones tendentes a mejorar su gestión, aunque no existe un departamento formal de gestión ambiental. (2) No existe vinculación entre el departamento de contabilidad y la gestión ambiental ejecutada. (3) El sistema contable no permita establecer los costos ambientales. Se concluyó lo siguiente: Se debe implantar una metodología que permita acumular los costos ambientales donde se imputen al costo del producto, actividad o periodo, y no segregarlos en un centro de costos ambientales separado.

Palabras clave: Aceite de palma, costos ambientales, gestión ambiental, impacto ambiental.

\section{Environmental Costs in the Process of Extracting Palm Oil. A Case Study}

\section{Resumen}

The objective of this investigation is to establish the factors that affect the formation of environmental costs in the palm oil extraction process at the processing plan Palmeras San Simón, in the Municipality of Colón, State of Zulia. The study is framed within financial accounting, on the internal costs base. The research is of a descriptive, field type; techniques utilized were the interview, direct observation and the survey. The following results were obtained: 1) The company knows about the principal

Recibido: 23-09-06 Aceptado: 11-03-08

Docente. UNESUR. Lic. en Contaduría Pública, - ULA. Magíster en Ciencias Contables - ULA. Cursante del Doctorado en Ciencias Sociales, Mención Gerencia - LUZ. Correo Electrónico: reinosad@gmail.com / dorisreinosa@cantv.net. 
effluents and the environmental impact and has established actions tending to improve their management, even though there is no formal department for environmental management. (2) No connections exist between the accounting department and the environmental management carried out. (3) The accounting system does not permit establishing environmental costs. Conclusions were the following: A methodology should be implanted that permits accumulating environmental costs, where they are attributed to the cost of the product, activity or period and not segregated in a separate environmental cost center.

Key words: Palm oil, environmental costs, environmental management, environmental impact.

\section{Introducción}

En los últimos años se ha presentando una sensibilidad especial respecto al tema del cuidado del entorno natural y el ambiente; todo esto como consecuencia de los problemas generados por los desechos de las industrias en su proceso productivo, y que desde el siglo pasado, han provocado innumerables pérdidas naturales y grandes riesgos a la salud y calidad de vida en la población.

El problema ambiental es tan serio que la primera evaluación de la contaminación global efectuada revela que más de 1.200 millones de hectáreas de tierra (equivale a la superficie combinada de China y la India) han sufrido una seria degradación en los últimos cuarenta y cinco años (World Resources Institute, 1996). Por esta razón, en el ámbito mundial se han creado normas ambientales que intentan resguardar el ambiente, tratando de disminuir al máximo la contaminación ambiental. En un principio las empresas vieron estas iniciativas de defensa del ambiente y la legislación ambiental, como un enemigo o elemento molesto en su gestión económica, luego tomaron una actitud de resignación y optaron por la adopción de programas de protección del ambiente. Recientemente, han empezado a comprometerse, aceptando su res- ponsabilidad y lanzándose voluntariamente a la defensa y protección del mismo.

En Venezuela, hasta el momento son pocos los sectores que se han ocupado de evaluar la gestión ambiental, pero están surgiendo nuevas leyes y disposiciones de tipo ambiental que obligan a las empresas u organizaciones a considerar el tema de la conservación del ambiente durante su proceso productivo, creando la necesidad de determinar cuál es el impacto ambiental generado por su actividad, de manera de desarrollar políticas que comprendan acciones preventivas y restauradoras que mejoren su gestión ambiental.

Gray et al. (1999), exponen que la contabilidad tiene un importante lugar en el proceso de mejorar la gestión ambiental, debido a que constituye la base para la toma de las decisiones gerenciales, y lo que es más importante ayuda a definir y medir el éxito de las acciones emprendidas por las organizaciones. En los actuales sistemas contables no se incluyen los aspectos ambientales, ocasionando que no se tomen en consideración en la toma de decisiones económicas. En ese sentido, los autores sostienen, que la contabilidad tiene un alto grado de responsabilidad en los actuales niveles de deterioro ambiental. 
En consecuencia, ha surgido una unión entre la ecología y la contabilidad, como una forma de dar respuesta a la problemática ambiental que se genera en las empresas. La ecología dirige sus esfuerzos a reconciliar el progreso y el ambiente, para disminuir el daño ecológico, asumiendo la responsabilidad del hombre y su papel en la protección del ambiente. La contabilidad aporta los medios para medir aquellos hechos económicos que afectan la entidad para brindar información relevante, confiable y oportuna. Con la incorporación de la materia ambiental en la contabilidad se busca contribuir con métodos para cuantificar, registrar e informar los daños causados al ambiente y las acciones preventivas o correctivas necesarias para evitarlos. Al resultado de esta unión entre ecología y contabilidad se le denomina Contabilidad Ambiental.

Dentro de las áreas más importantes de la Contabilidad Ambiental se encuentran los Costos Ambientales entendidos como la estimación del costo global que supone la mitigación de los daños ambientales que se hayan causado a partir de un producto, actividad o proceso. El problema es que la mayoría de las empresas no están preparadas para brindar ese tipo de información, puesto que los sistemas tradicionales de contabilidad ocultan los costos ambientales en las cuentas de gastos generales. En la medida que la empresa identifique estos costos por separado, tendrán la posibilidad de analizar las ventajas financieras de aplicar prácticas de conservación ambiental.

En Venezuela, uno de los rubros agrícolas con más auge es la palma aceitera, la población de Santa Bárbara ubi- cada al sur del Lago de Maracaibo, se proyecta como un gran centro de producción, donde se ubican aproximadamente 20.000 has cultivadas de las 30.000 has que existen en todo el país (Moreno 2001). Este tipo de palma se caracteriza por ser el cultivo oleaginoso que produce mayor cantidad de aceite por unidad de superficie, y genera gran cantidad de productos, que son utilizados en la alimentación y la industria. Además, la palma aceitera, se considera un rubro bandera dentro de las políticas agrícolas del Estado y su cultivo esta destinado a cubrir el déficit de grasas y aceite vegetal en el país, además de proyectarse con un gran mercado para exportación (Moreno, 2001).

En ese sentido, Bautista (2001), presidente de la Corporación para el Desarrollo del Zulia (Corpozulia) informó sobre un acuerdo que se firmo entre la corporación y la empresa privada para sembrar 200 hectáreas de palma aceitera adicionales a las ya existentes, con una inversión de 800 millones de dólares. Inversión que convertirá a esta entidad en una potencia latinoamericana. Todo esto creó la necesidad del establecimiento en la zona de plantas procesadoras que permitan la extracción del aceite del fruto, convirtiéndose en una nueva fuente de empleos y desarrollo económico. Entre estas iniciativas se pueden contar hasta el momento el establecimiento de dos plantas procesadoras de palma aceitera en el Municipio Colón del Estado Zulia.

El procesamiento industrial del fruto de la palma de aceite genera desechos o residuos, que tarde o temprano encuentran la forma de regresar al entorno natural. De acuerdo como se manipulen estos residuos pueden conducir a la contami- 
nación o a la degradación del ambiente. Por tanto, es necesario crear medidas para la prevención de la contaminación, y es en esa oportunidad que la contabilidad empieza a jugar un papel importante, al brindar los mecanismos necesarios para determinar los costos de las actividades destinadas a prevenir y mitigar el daño ambiental, convirtiéndose en una herramienta indispensable para la toma de decisiones.

Debido a que las empresas procesadoras de aceite de palma en el Municipio Colon se instalaron hace poco tiempo, en la investigación que precede este articulo se estableció como objetivo de identificar de los factores que inciden en la conformación de los costos ambientales en el proceso de extracción del aceite de palma, permitiendo establecer los impactos ambientales del proceso productivo, así como, las medidas ambientales emprendidas por la empresa, para luego asociarlas a algún tipo de costo ambiental, contribuyendo a cuantificar los esfuerzos realizados en cuanto a la prevención y control de la contaminación y a medir los resultados de su gestión.

En cuanto a la metodología, realizo una investigación de tipo descriptiva, de campo, de acuerdo con las estrategias a usar por el investigador para la recolección de los datos. Se siguieron los siguientes pasos: (a) revisar los aspectos teóricos relacionados, (b) identificar los aspectos contables de la empresa, (c) describir el proceso productivo, (d) identificar los aspectos ambientales factibles de ser contabilizados. Todo ello con el propósito de establecer los factores que inciden en la conformación de los costos ambientales en el proceso de extracción del aceite de palma. Se eligió como unidad de estudio la Planta Procesadora Palmeras San Simón, ubicada en la población de Santa Bárbara, Municipio CoIon, Estado Zulia. Las técnicas utilizadas para la recolección de la información fueron: la entrevista, el cuestionario y la observación directa.

\section{Responsabilidad y gestión ambiental empresarial}

Las actividades económicas realizadas por las empresas generan daños y perturbaciones en el ambiente. En el pasado muy pocas empresas se detenían a medir el impacto ambiental que generaba su gestión económica, ni se pensaba en desarrollar programas que minimizaran los daños causados al ambiente, se sacrificaba el ambiente en búsqueda de la optimización de los beneficios. Actualmente, las empresas están tomando conciencia de su responsabilidad en materia ambiental y esta conciencia ecológica ha sido motivada por las presiones de los gobiernos, de la opinión pública representada por grupos ambientalistas y la posibilidad de mejorar su imagen corporativa.

Según Rodríguez y Ricart (1998), de acuerdo a la actitud de las empresas ante la problemática ambiental podrían clasificarse en: (a) Reactivas, que consideran el asunto ambiental bajo un enfoque meramente técnico que requiere la creación de departamentos competentes para manejarlo y donde la alta dirección solo se involucra cuando no hay otra opción. (b) Proactivas, consideran que el problema ambiental afecta toda la organización, lo incorporan en sus procesos de definición estratégica y es parte de la res- 
ponsabilidad de la dirección general; para estas empresas, el ambiente no es un problema sino un reto y una oportunidad sea cual sea la industria en que compitan.

Estos autores exponen que la situación ambiental a llevado a las empresas afrontar un reto de una magnitud sin precedentes; ya que la sociedad les exige la creación de riqueza para satisfacer las necesidades no sólo de los países más desarrollados sino también de la creciente población de los países en vías de desarrollo. Por otra parte, se les exige que disminuyan el uso de recursos y la producción de todo tipo de desechos, debido a que la Tierra es un sistema cerrado, con riquezas naturales limitadas y con una capacidad de carga de contaminación también limitada, situación que a conllevado a que en los últimos años las empresas a nivel internacional le han concedido un espacio de gran importancia al problema ambiental.

En opinión de Epsteín (2000) toda la situación descrita a originado que las empresas integren las preocupaciones ambientales a sus decisiones administrativas en todos los niveles, y la tendencia es más a la iniciativa que a la reacción; y se aprecia una orientación más a la planeación que al mero cumplimiento de la legislación. En conclusión expone que en la actualidad dentro de las organizaciones están naciendo iniciativas concretas y espontáneas tendentes a mejorar la gestión ambiental, sin estar sometidas a presiones y sanciones por parte de la legislación.

Sin embargo, autores como Rodríguez y Yardin (1999) exponen que resultaría muy ingenuo suponer que las empresas ignoran el daño ambiental que ge- neran, por lo que se debe considerar que esas conductas socialmente indeseables sólo podrán ser modificadas a partir de una legislación fuertemente sancionadora y/o de grandes campañas de opinión que pongan a la empresa en riesgo de perder importantes sectores de mercado por su desprestigio social.

Ante tal dualidad de opiniones es necesario fijar una posición, en tal sentido, consideramos que el escenario planteado por autores como Epsteín (2000) y Rodríguez y Ricart (1998) se corresponde al comportamiento empresarial en los países desarrollados. En los países en vías de desarrollo, como Venezuela, que a pesar de ser uno de los pioneros en Latinoamérica en promulgar leyes de protección ambiental, se está lejos de alcanzar un verdadero compromiso ambiental del sector empresarial, del Estado y la población en general.

\section{Contabilidad ambiental}

La Fundación Forum Ambiental, Agencia Europea de Medio Ambiente (1999: 7), definió la contabilidad ambiental como la generación, análisis y utilización de información financiera y no financiera destinada a integrar las políticas económicas y ambientales para construir una empresa compatible con el desarrollo sostenible.

Para Bravo (1997) existen tres tipos de la Contabilidad Ambiental: (1) Contabilidad de la Nación (cuentas nacionales); (2) Contabilidad Financiera, referida a la estimación e información de las responsabilidades ambientales y los costos desde un punto de vista financiero, ofrece información a usuarios externos como el go- 
bierno, inversionistas y a la opinión pública en general; (3) Contabilidad Gerencial o Contabilidad Administrativa, entendida como el proceso de identificación, recolección y análisis de información para uso interno, es decir, al servicio de las necesidades de la administración.

La presente investigación se enmarca dentro de los límites de la contabilidad financiera, entendida según Ablan y Méndez (2004) como un sistema de información a través del cual se recopila, mide, e informa los resultados de las operaciones de la empresa tomando en consideración su relación con el ambiente, con la finalidad de homogeneizar la información financiera y atendiendo los Principios de Contabilidad Generalmente Aceptados, en el caso Venezolano se deben elaborar atendiendo las Normas Internacionales de Contabilidad NIC's. Estos autores resaltan que esta disciplina apenas comienza considerar el efecto del ambiente sobre la información financiera y no existe normativa específica al respecto. Hasta ahora la contabilidad ambiental es el resultado de iniciativas voluntarias.

Sin embargo, debido a la relevancia de la situación ambiental en ámbito mundial y la importancia que ha adquirido para las empresas, se considera que estas deben reflejar los efectos de su gestión ambiental en sus estados financieros, ya que la información contenida en la contabilidad, no solo interesa a los accionistas, sino también a todos aquellos que se mueven en el entorno de la empresa.

Pero, lograr una verdadera contabilidad ambiental requiere un profundo cambio en su concepción actual, al respecto, Ariza (2000) sostiene que a partir de los datos contables se instauran normas que gobiernan la vida de las organizaciones y cada vez en más facetas el futuro de la sociedad. En la actualidad, la contabilidad se encuentra inmersa en la lógica del lucro y deberá emprender esfuerzos teóricos y epistemológicos para contribuir a un verdadero desarrollo sostenible.

Gray et al. (1999) enuncian algunos aspectos que deberá abarcar la contabilidad ambiental:

- Identificación separada de los costos e ingresos relacionados con el ambiente, dentro de los sistemas contables tradicionales.

- Establecer iniciativas para aminorar los efectos ambientales existentes en la práctica contable tradicional.

- Idear nuevas formas de sistemas contables, sistemas de información y sistemas de control, financieros y no financieros, para fomentar decisiones administrativas ambientalmente benignas.

- Desarrollar nuevas formas de medición de desempeño, información y valoración para propósitos tanto internos como externos.

- Identificar y rectificar áreas donde existan conflictos entre los criterios convencionales (financieros) y ambientales.

- Experimentar formas para valorar la sostenibilidad e incorporarla a la organización.

\section{Costos ambientales}

Una de las áreas de la contabilidad ambiental con más proyección son los 
costos ambientales, que consisten en la valoración de la aplicación racional de los factores ambientales, necesarios para la obtención de un producto, la realización de un proceso o la prestación de un servicio, y la asimilación por el entorno natural de los desechos de las actividades de producción y consumo del ser humano (Scavone et al,1999). Es decir, son todos los sacrificios debidamente valorados de:

- Las acciones destinadas a prevenir, reducir o resaltar los daños ambientales que son consecuencia de las actividades de explotación.

- Las acciones relacionadas con la conservación de los recursos naturales y no renovables.

- La aplicación racional de los factores ambientales productivos.

En concordancia con lo anterior, Ariza (2000) proporciona algunos ejemplos de rubros identificados como costos ambientales: insumos y/o materias primas no contaminantes, y cuotas de depreciación de maquinaria o tecnología ambientalizada.

\subsection{Naturaleza de los costos ambientales}

Las empresas incurren en costos ambientales por tres motivos distintos (Seoánes, 1999): (a) legales, (b) sociales, (c) de mercado. Los legales derivan de las normas ambientales emitidas por los entes de control. Los sociales y culturales derivan de las expectativas de la sociedad y la cultura que opera en la empresa. Los de mercado derivan de la presión que ejercen los consumidores al preferir productos que cumplen con normas ambientales.

Tipos de costos ambientales: Existen diversas clasificaciones de costos ambientales, que varían de acuerdo a los distintos modelos de costos ambientales existentes, Spallarosa (2000) propone una de las más usadas:

- Costos de Prevención. Son aquellos destinados a eliminar potenciales causas de impactos ambientales negativos. Por ejemplo, el rediseño de un proceso o la sustitución de materiales.

- Costos de Evaluación. Son dirigidos a medir y monitorear las fuentes potenciales de daños ambientales. Por ejemplo, auditorias ambientales, información por suministrar a los entes de control, monitoreo de emisiones.

- Costos de Control. Son aquellos encaminados a contener sustancias peligrosas que son utilizadas o producidas. Por ejemplo, plantas de tratamiento o tanques reforzados para almacenar productos químicos.

- Costos de Fracaso. Son los destinados a remediar los daños ambientales ocasionados.

Para efectos de la presente investigación los tipos de costos ambientales se sintetizarán a tres: costos de prevención, de control y de mitigación o fracaso.

\subsection{Alcance de los costos ambientales}

Spallarossa (2000) explica que los Costos Ambientales pueden considerar sólo los costos internos o también puede incluir los costos externos (sociales o ex- 
ternalidades). Los costos internos que se derivan de las actividades de la empresa en su interacción con el ambiente, es decir, son el resultado de la armonización del proceso productivo con ambiente, y sólo impactan el resultado contable de la empresa. Los costos externos surgen del impacto ambiental como consecuencia del accionar de los agentes contaminantes, estos afectan a los individuos, a la sociedad, y al ambiente externo a la empresa.

\subsection{Costos internos vs. Costos externos}

Hasta el momento no esta definido si los costos ambientales deben incluir únicamente los costos internos o si adicionalmente deben incluirse los costos externos o sociales, en cuanto a ese punto existen posiciones contrarias bien marcadas, quienes afirman que deben incluir los costos externos o sociales están fuertemente influenciados por las nuevas tendencias de la contabilidad social, y por el contrario quienes sostienen que solo deben incluir los costos internos están basados en los principios de la contabilidad financiera tradicional.

Para aclarar el punto, a continuación se plantea la opinión de Rodríguez y Yardin (1999:7) expuesta ante XXII Congreso Argentino de Profesores Universitarios de Costos: Opinamos que ningún "costo externo" debe ser registrado por la empresa y mucho menos incorporado como tal al costo de producto o del período. En efecto, si existiera alguna resolución concreta del sector público o una decisión interna de la empresa que determinara la necesidad de la adopción de medidas preventivas o correctivas, estaría- mos ante la presencia de un "costo interno" -independientemente de su intencionalidad- con un criterio de medición, registración y exposición absolutamente ordinario. Pero si no existe ninguna legislación punitiva ni ninguna decisión interna en ese sentido, proponer la registración e incorporación al costo del producto o del período del mero reconocimiento de esos "costos externos" o "externalidades" parece ajeno a toda lógica contable o empresarial.

Para reforzar la idea expuesta, Rodríguez y Yardin (1999:7) citaron el siguiente ejemplo: "La idea de la incorporación del "costo externo" tal como la hemos encontrado expuesta, parece responder a un razonamiento de este tipo: El costo de una campera de cuero de carpincho no es solamente el costo de comprar la piel, curtirla y procesarla, sino que debe tomar en consideración el efecto medioambiental que produce el hecho de que se trate del cuero de una especie en peligro de extinción. Expresando nuestra coincidencia total con quienes repudian la conducta de todos los que intervienen en este proceso -desde el que caza el carpincho hasta el que compra la campera- y que la desaparición de una especie animal significa una importante pérdida para toda la humanidad, creo que postular que una empresa que desarrolle esta actividad incorpore al costo de su producto el daño ecológico que genera sería un verdadero disparate (independientemente del debate bizantino que plantearía su medición)".

En atención a lo expuesto, consideramos desde la concepción de costos internos, que para la empresa solo es posible evaluar el efecto de las acciones am- 
bientales o de la inacción empresarial sobre el resultado financiero y no sobre el impacto que pueda tener sobre paisaje urbano, el ecosistema o la sociedad. En consecuencia, la presente investigación se ha enmarcado dentro de la corriente de costos internos y no se abordaron los costos externos o sociales que pudieran derivarse de la actividad productiva de la empresa objeto de estudio. Al asumir esta posición no desmerecemos la corriente de los costos externos, pero, consideramos que en la actualidad no existen las herramientas que permitan medir, registrar e informar en los estados financieros el impacto ambiental que se genera de la actividad empresarial y afecta a la sociedad o el ecosistema.

En cuanto al impacto ambiental que incide en la sociedad y el ecosistema, los autores Rodríguez y Yardin (1999) explican que desde el enfoque de la contabilidad de la nación resulta necesario medir y registrar los costos y beneficios medioambientales para lograr una correcta evaluación de los bienes de la nación. Por lo que resultaría deseable poder contabilizar el costo de la degradación del ambiente originada en acontecimientos naturales o en acciones humanas y los beneficios obtenidos mediante acciones de conservación o reparación. Un procedimiento apto sería la incorporación de cuentas en la Contabilidad Nacional que reflejaran estas disminuciones o incrementos de la calidad ambiental.

\section{Contabilidad Social}

Es una nueva tendencia enmarcada dentro de la responsabilidad social empresarial que comienza a influenciar la contabilidad, al respecto Marín (s/f) realiza una expone que la contabilidad social replantea el sentido de la información contable y reivindica el valor de uso de la contabilidad en cuanto se satisface una necesidad social. La concepto de contabilidad social asume que la información producida debe investirse de objetividad e imparcialidad, para permitirle a la comunidad saber en como las actividades económicas afectan o benefician a los grupos sociales de su medio geográfico circundante, con el fin de establecer la concordancia existente entre las expectativas reales de la colectividad y las actuaciones de la empresa, abogando por la rendición de cuentas de las acciones desarrolladas por la unidad productiva.

Consideramos que esta nueva tendencia en contabilidad a ejercido una fuerte influencia sobre algunos autores que trabajan en el área de contabilidad ambiental, asumiendo que la misma puede brindar grandes soluciones desde el punto de vista de social, apartándose de la línea de la contabilidad financiera tradicional.

\section{Descripción del proceso productivo y tipos de efluentes}

El procesamiento industrial del fruto de la palma de aceite origina tres productos: el aceite de palma, el aceite de palmiste o almendra y la torta o harina de palmiste. Para obtenerlos se requieren dos procesos principales y uno auxiliar. A partir de las entrevistas y la observación realizada a continuación se presenta una breve descripción de cada uno de estos procesos y se identifican el tipo de efluente que origina. 


\subsection{Extracción del aceite de palma}

La extracción del aceite de palma (Diagrama 1) es el principal proceso de esta empresa, y consta de las siguientes fases:
- Recepción de la fruta. Consiste en el pesaje del camión cargado de fruta al entrar a las instalaciones de la planta, lo que permite tener un control diario de la materia prima disponible. Efluente $\mathrm{N}^{\circ} 1$ : Impurezas de las frutas.

\section{Diagrama 1}

Proceso de extracción de aceite de palma y sus efluentes

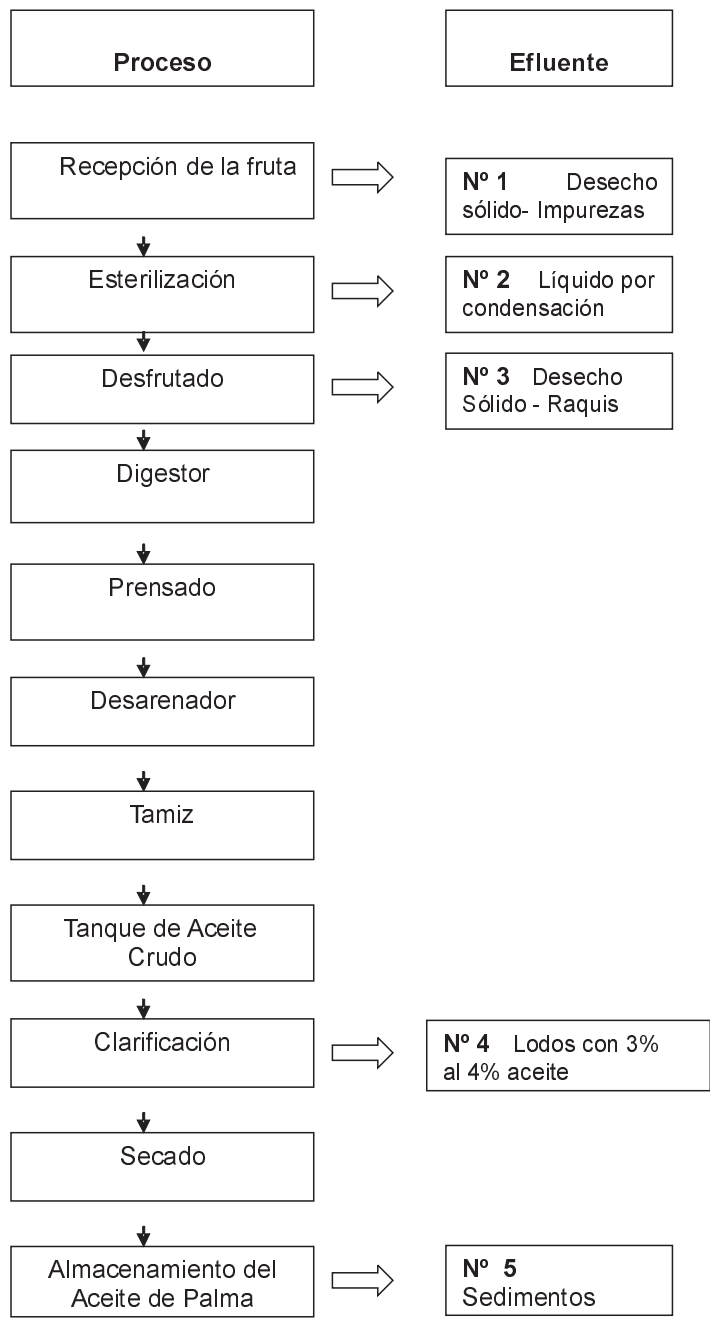

Fuente: Elaboración propia (2006). 
- Esterilización. Es el sometimiento de la fruta a condiciones de temperatura y presión de vapor saturado durante un periodo de 75 minutos. En el proceso la fruta cargada en vagones se ingresa al esterilizador donde la temperatura debe estar entre $140^{\circ}$ y $150^{\circ}$ centígrados. Efluente $\mathrm{N}^{\circ} 2$ : Líquidos por condensación.

- Desfrutado. Es la separación mecánica de los frutos presentes en los racimos mediante un tambor desfrutador rotatorio. La fruta esterilizada es Ilevada a la desfrutadora que realiza la separación de los frutos de las raquis mediante golpes continuos. Efluente $N^{\circ} 3$ Raquis vacías.

- Prensado. Comprende en primer lugar en la digestión que consiste en macerar la fruta a una temperatura de $90^{\circ} \mathrm{C}$. En segundo lugar el prensado, donde se extrae mecánicamente por presión el aceite contenido en el mesocarpio de la fruta, requiere adicionar agua caliente con el fin de ayudar a arrastrar el aceite y mantener la temperatura constante hasta la clarificación. De esta etapa se originan dos corrientes, una líquida que pasa a clarificación y una sólida que pasa al desfibrado.

- Clarificación. Consiste en retirar el agua y las impurezas lodosas del aceite, mediante el filtrado, la decantación y centrifugación, buscado alcanzar altos rendimientos de aceite crudo con un mínimo de impurezas. Efluente $\mathrm{N}^{\circ} 4$ Lodos residuales que salen del clarificador.

- Almacenamiento. Una vez que el aceite alcanza los niveles de pureza requerido es enviado a los tanques de almacenamiento. Efluente $\mathrm{N}^{\circ} 5$ Sedimentos del tanque de almacenamiento.

\subsection{Extracción del aceite y harina de palmiste}

La extracción del aceite de palma y harina de palmiste (Diagrama 2) consta de las siguientes fases:

- Desfibrado. De la fruta prensada se origina una pasta compuesta por fibras y nueces, el desfibrado consiste en la separación de los dos elementos aprovechando la diferencia de densidad. Efluente $\mathrm{N}^{\circ} 6$ Fibra de palma, es almacenada y utilizada para combustible de la caldera.

- Palmistería. Consiste en la obtención de la almendra o palmiste. Las nueces se rompen y pasan a un tambor despericarpiador donde se separa la cáscara de las almendras. Las almendras limpias pasan a un silo secador donde se les inyecta aire caliente. Efluente $\mathrm{N}^{\circ} 7$ Cáscara de la nuez, que es utilizada como combustible para la caldera. Efluente $N^{\circ} 8 \mathrm{Li}-$ quido originado por el secado de la nuez.

- Extracción de aceite de almendra o palmiste. El objetivo del proceso es extraer el aceite de la almendra. En el proceso la almendra es triturada y prensada hasta obtener el aceite que se tamiza para retirar las impurezas presentes. La parte sólida se denomina torta o harina de palmiste que es empacada en bultos para ser vendida como materia prima para la elabora- 


\section{Diagrama 2 \\ Proceso de extracción de aceite de almendra palma y sus efluentes}

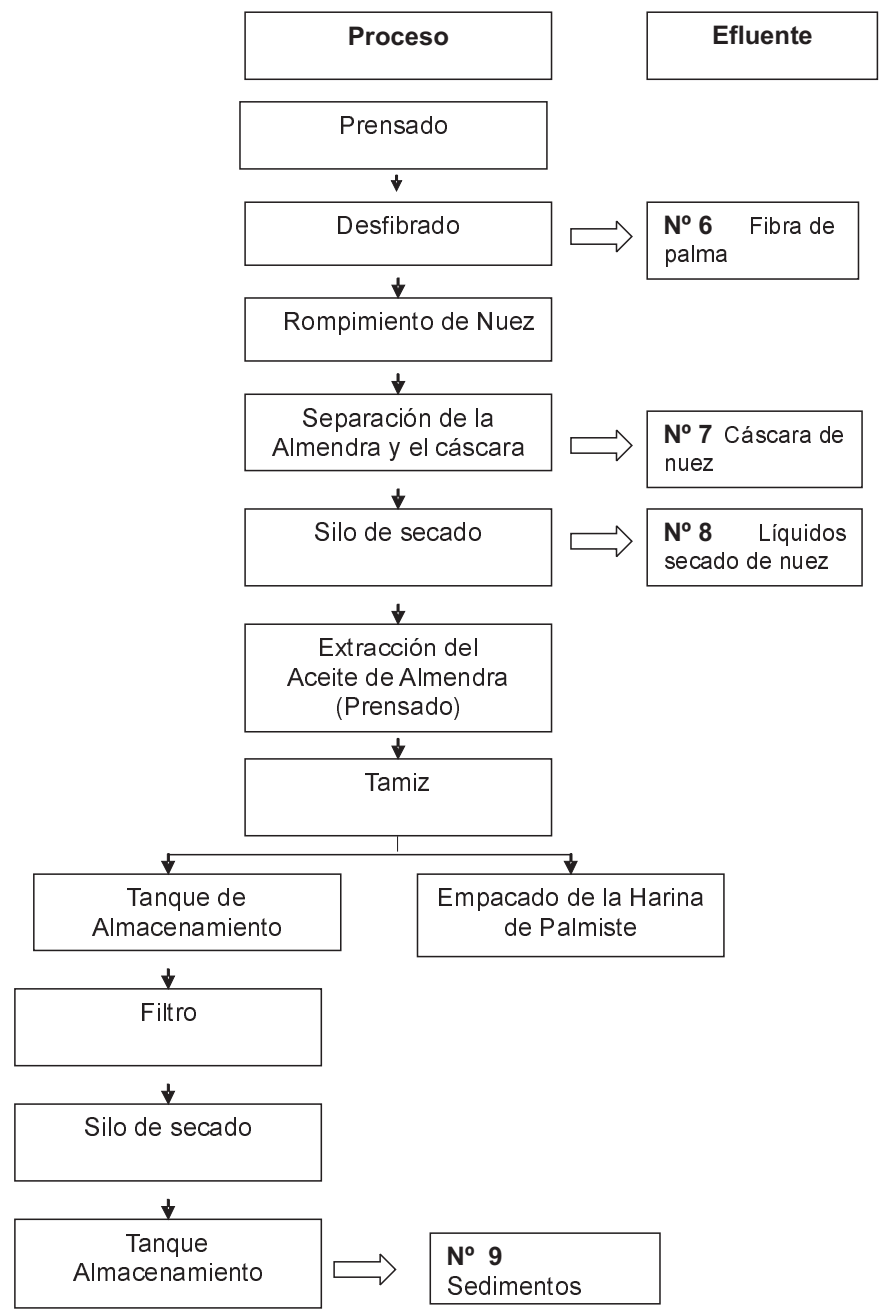

Fuente: Elaboración propia (2006).

ción de alimento concentrado para ganado.

- Almacenamiento. El aceite de palmiste pasa a los a los tanques de almacenamiento. Efluente № 9 Sedimentos del tanque de almacenamiento.

\subsection{Generación de vapor}

La generación de vapor (Diagrama 3) es necesaria como fuente de calor para los equipos de la planta, la caldera utiliza como combustible la fibra y la cás- 


\section{Diagrama 3 \\ Utilización de Vapor}

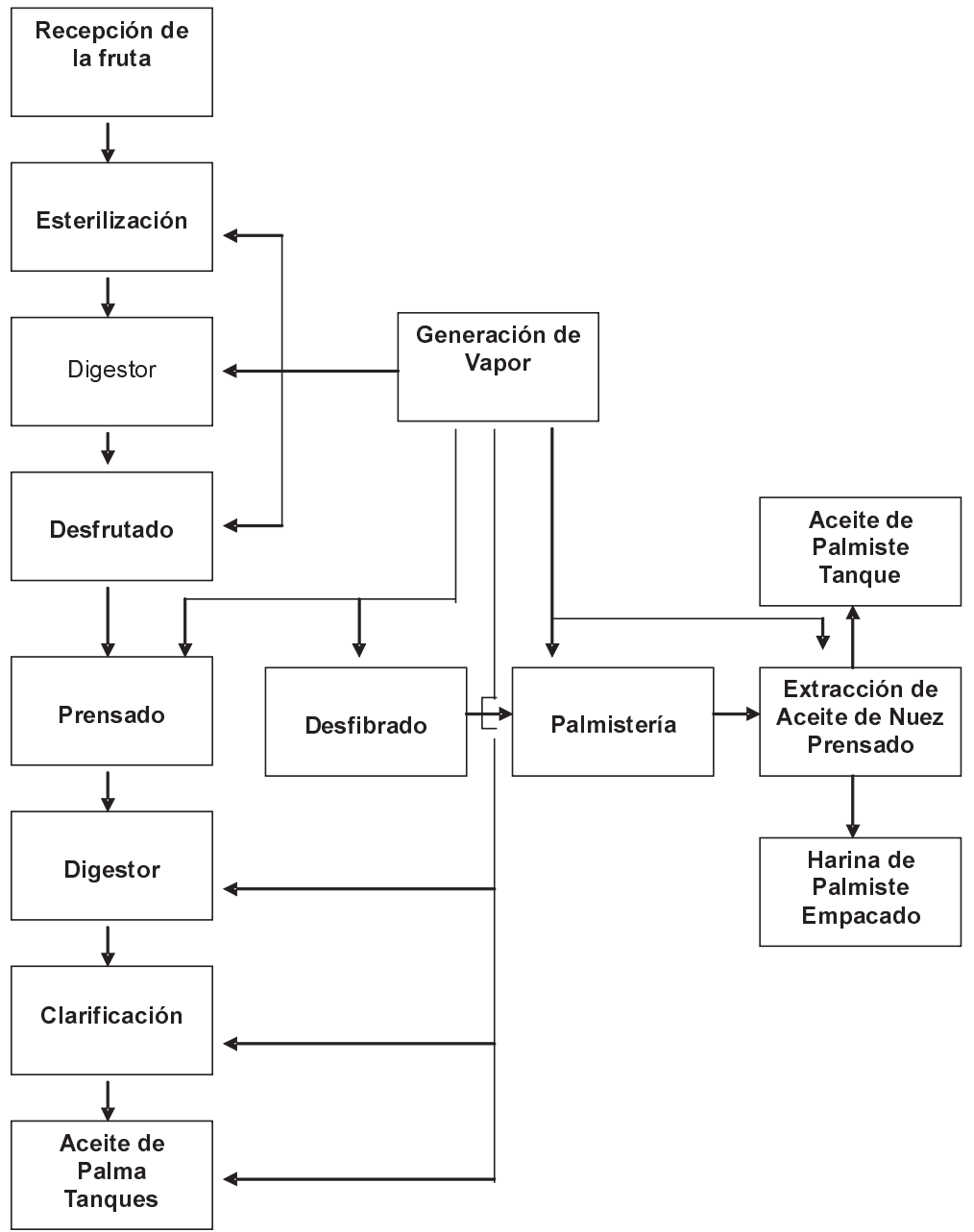

Fuente: Elaboración propia (2006).

cara generada en el proceso productivo. La calidad del vapor es del tipo saturado y para controlar la emisión de partículas por la chimenea se emplea un sistema de recolección de cenizas, compuesto por un ventilador, ciclón y esclusa. Efluente No 10 Ceniza de la caldera.

\section{Identificación de los residuos y su destino final}

En el Cuadro 1 se establecen las etapas del proceso productivo, los efluentes y el destino que tienen los mismos, con el propósito de establecer 


\section{Cuadro 1}

Residuos originados en el proceso productivo y su destino final

\begin{tabular}{|c|c|c|}
\hline Etapas del proceso & Efluentes & Destino o tratamiento \\
\hline Recepción de la fruta & $\mathbf{N}^{\circ} 1$ Impureza de la fruta & Plantaciones, como abono. \\
\hline Esterilización & $\begin{array}{l}\text { № } 2 \text { Líquidos por } \\
\text { condensación }\end{array}$ & $\begin{array}{l}\text { Planta de tratamiento de } \\
\text { aguas }\end{array}$ \\
\hline Desfrutado & № 3 Raquis vacías & $\begin{array}{l}\text { Se almacenan a campo } \\
\text { abierto y luego se envían a } \\
\text { las plantaciones como control } \\
\text { de malezas }\end{array}$ \\
\hline Prensado & - & - \\
\hline Clarificación & $\mathbf{N}^{\circ} \mathbf{4}$ Aguas y lodos residuales & $\begin{array}{l}\text { Planta de tratamiento de } \\
\text { aguas }\end{array}$ \\
\hline Desfibrado & $N^{\circ} 6$ Fibra de la palma & Combustible para la caldera \\
\hline \multirow[t]{2}{*}{ Palmistería } & № 7 Cáscara de la nuez & Combustible para la caldera \\
\hline & $\begin{array}{l}\text { No } 8 \text { Líquido por secado de la } \\
\text { nuez }\end{array}$ & $\begin{array}{l}\text { Planta de tratamiento de } \\
\text { aguas }\end{array}$ \\
\hline Extracción del aceite de nuez & - & - \\
\hline $\begin{array}{l}\text { Almacenamiento del aceite } \\
\text { de palma y nuez }\end{array}$ & $\begin{array}{l}\mathbf{N}^{\circ} 5 \text { y } \mathbf{N}^{\circ} 9 \text { Sedimentos del } \\
\text { tanque }\end{array}$ & $\begin{array}{l}\text { Planta de tratamiento de } \\
\text { aguas }\end{array}$ \\
\hline $\begin{array}{l}\text { Empacado de la torta o } \\
\text { harina de nuez }\end{array}$ & - & - \\
\hline Generación de vapor & $\mathbf{N}^{\circ} 10$ Cenizas & $\begin{array}{l}\text { Se almacena en campo } \\
\text { abierto }\end{array}$ \\
\hline
\end{tabular}

Fuente: Elaboración propia.

aquellas áreas donde la empresa a iniciado acciones tendentes a prevenir, controlar y mitigar el daño ambiental, acciones que al ser clasificadas, monitoreadas y cuantificas forman parte del costo ambiental. Así mismo, permite visualizar aquellas etapas donde se presentan debilidades en la gestión ambiental. Adicionalmente, se incluye el Diagrama 4 donde se muestra el recorrido de los efluentes líquidos hasta el sistema de tratamiento de aguas.

\section{Efectos ambientales potenciales y medidas de prevención y protección}

En el Cuadro 2 se presentan los tipos de contaminación que se derivan del proceso productivo y su relación directa con el efecto ambiental potencial, se han desincorporado los tipos de contaminación para los que la empresa tiene mecanismos de control. Identificar los efectos potenciales sobre el ambiente se considera de relevancia pues a partir de ella se pueden iniciar acciones de prevención 


\section{Diagrama 4 \\ Efluentes Líquidos}
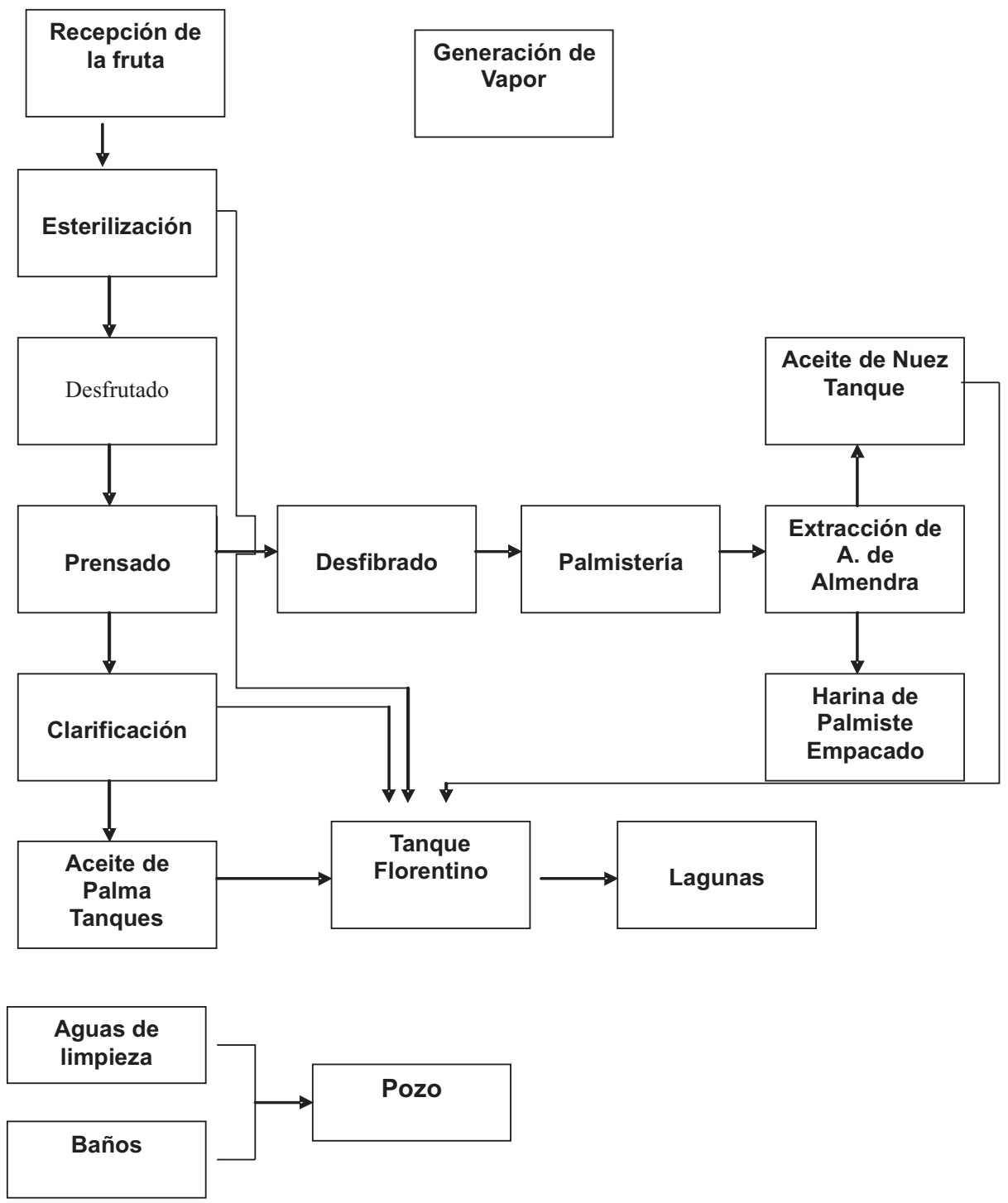

Fuente: Elaboración propia (2006). 


\section{Cuadro 2}

Efectos ambientales potenciales

\begin{tabular}{|c|c|c|}
\hline Etapa del proceso & Tipo de contaminación & Efecto \\
\hline Recepción de la fruta & - Ruido y Polvo & Afectación de la salud \\
\hline Esterilización & - Ruido, Olor y Polvo & Afectación de la salud \\
\hline Desfrutado & - Polvo & Afectación de la salud \\
\hline Prensado & - Ruido & Afectación de la salud \\
\hline Clarificación & - Ruido & Afectación de la salud \\
\hline Desfibrado & - Ruido & Afectación de la salud \\
\hline Palmistería & - Ruido y Polvo & Afectación de la salud \\
\hline Extracción del aceite de nuez & - & - \\
\hline $\begin{array}{l}\text { Almacenamiento del aceite } \\
\text { de palma y nuez }\end{array}$ & - & - \\
\hline \multirow{2}{*}{$\begin{array}{l}\text { Empacado de la torta o } \\
\text { harina de nuez }\end{array}$} & - Polvo & Afectación de la salud \\
\hline & - Riesgo de accidentes & \\
\hline \multirow{4}{*}{ Generación de vapor } & - Material particulado (ceniza) & Contaminación atmosférica \\
\hline & - Polvo y Ruido & Afectación de la salud \\
\hline & $\begin{array}{l}\text { - Riesgos en la manipulación } \\
\text { del combustible }\end{array}$ & Afectación de la salud \\
\hline & $\begin{array}{l}\text { - Escurrimiento de sólidos des- } \\
\text { de el centro de disposición de } \\
\text { cenizas }\end{array}$ & Contaminación de aguas \\
\hline
\end{tabular}

Fuente: Elaboración propia.

que estarían relacionadas directamente con la conformación del costo ambiental.

El estudio realizado se complementa con el Cuadro 3 donde se sugieren medidas de prevención para los efectos ambientales potenciales ya identificados. Algunas de las medidas sugeridas ya se han implementado en la empresa, tal es el caso de la planta de tratamiento, pero existen otras áreas como el ambiente de trabajo interno de la empresa donde existen fallas en la protección individual de los trabajadores, y es necesario adoptar mejores medidas de protección.

\section{Elementos que inciden en la conformación del costo ambiental}

En el Cuadro 4 se presenta ejemplo ilustrativo de cómo conformar el costo ambiental a partir de la identificación del tipo de contaminación, para tal efecto se ha elegido una clasificación de costos ambientales que se considera general y fácil de visualizar por aquellos profesionales de la Contaduría Publica que se inician en este ámbito de trabajo, se basa e 


\section{Cuadro 3 \\ Efectos ambientales y medidas de prevención y protección}

\begin{tabular}{|c|c|c|}
\hline Tipo de contaminación & Efecto & Prevención \\
\hline Ruido & Afectación de la salud & $\begin{array}{l}\text { Protección individual: casco, } \\
\text { orejeras, rotación de puestos } \\
\text { de trabajo. }\end{array}$ \\
\hline Polvo & Afectación de la salud & $\begin{array}{l}\text { Protección individual: uso de } \\
\text { mascarillas }\end{array}$ \\
\hline Líquidos & $\begin{array}{l}\text { Contaminación del agua y } \\
\text { suelo }\end{array}$ & $\begin{array}{l}\text { Planta de tratamiento de aguas } \\
\text { residuales. }\end{array}$ \\
\hline Desecho sólido & Degradación del paisaje & Disposición y recuperación* \\
\hline Olores & $\begin{array}{l}\text { Afectación de la salud } \\
\text { Contaminación del aire }\end{array}$ & $\begin{array}{l}\text { Protección individual: uso de } \\
\text { mascarillas } \\
\text { Cortinas vegetales }\end{array}$ \\
\hline
\end{tabular}

Fuente: Elaboración propia.

* Recuperación es la utilización del residuo generado en otro proceso distinto del que lo ha producido, este se podrá introducir en el nuevo proceso directamente o mediante algún tratamiento previo.

\section{Cuadro 4 \\ Elementos que inciden en la conformación del costo ambiental}

Tipo de contaminación

Líquidos

Sólidos

Material Liberado al Aire

Fuente: Elaboración propia.
Costo ambiental

- Prevención: Monitoreo de niveles de ruido

- Control: Mantenimiento y ajuste de la Maquinaria

- Mitigación: Cascos y orejeras

- Prevención:

- Control:

- Mitigación: Mascarillas y Cortinas Vegetales

- Prevención:

- Control: Depreciación de la planta de tratamiento

- Mitigación: Monitoreo de los niveles del agua

- Prevención:

- Control: Centro de disposición

- Mitigación: Programas para recuperación de la materia

- Prevención: Monitoreo de niveles de ceniza liberada

- Control: Depreciación del ciclón

- Mitigación: 
una simplificación de la clasificación sugerida por Spallarosa (2000):

1. Costos de Prevención. Son aquellos destinados a eliminar potenciales causas de impactos ambientales negativos. Por ejemplo el rediseño de un proceso o la sustitución de materiales.

2. Costos de Control. Son aquellos encaminados a contener sustancias peligrosas que son utilizadas o producidas. Por ejemplo, plantas de tratamiento o tanques reforzados para almacenar productos químicos.

3. Costos de Mitigación o Fracaso. Son los destinados a remediar los daños ambientales ocasionados.

En este caso se presenta un ejemplo de elementos que conforman el costo ambiental en función del tipo de contaminación (Cuadro 4).

\section{Conclusiones}

Como se enuncio el propósito de este artículo es establecer los factores que inciden en la conformación de los costos ambientales en el proceso de extracción del aceite de palma, y para tal fin se trazaron algunas rutas a seguir: (a) descripción del proceso productivo, (b) identificación de los aspectos contables de la empresa, y (c) identificación de los aspectos ambientales factibles de ser contabilizados. Estas servirán de estructura para esbozar las conclusiones.

El proceso productivo de la empresa puede dividirse en dos procesos principales. (a) la extracción del aceite de palma y (b) la extracción del aceite de almendra y harina de palmiste. Adicionalmente, existe un tercer proceso indispensable para desarrollar los dos primeros, la ge- neración de vapor. De estos procesos se identificaron diversos efluentes, tales como: vertidos líquidos, residuos sólidos y emisiones al aire. Dichos efluentes pueden causar degradación al ambiente, en ese sentido, se constato que se han realizado esfuerzos por disminuir el impacto ambiental. Adicionalmente, el proceso productivo genera una serie de contaminantes (ruido, olores, vibraciones) que son potencialmente dañinos para la salud de los trabajadores y que no están atendidos correctamente, en consecuencia, se requiere implementar mayores medidas de seguridad.

En el estudio realizado se determino que no existe un Departamento de Gestión Ambiental pero puede apreciarse que existe una disposición para mejorar la gestión ambiental de la empresa, y para ello se han iniciado algunas medidas destinadas a la armonización de las actividades de la empresa con el ambiente, tales como, la implementación de una planta de tratamiento de aguas residuales, el ciclón para recolectar ceniza y la utilización de los desechos sólidos en elaboración de compost y como fuente de energía para general vapor. Estas iniciativas junto a otras pueden llegar a ser articuladas en un plan de gestión ambiental, con objetivos bien definidos.

Todo lo anterior pone en evidencia que es posible que existan iniciativas voluntarias en el sector empresarial para armonizar la actividad productiva con el medio ambiente, sin embargo, es un proceso que requiere la participación de la Gerencia, la incorporación de ambiente en la planificación y toma de decisiones, la creación de estructuras especializadas e inversiones de capital significativas. 
Durante la investigación se constato la desvinculación del departamento de contabilidad con la situación ambiental de la empresa, así como, el desconocimiento sobre conceptos como contabilidad ambiental, costos ambientales y normas técnicas y legislación relacionada con contabilidad ambiental; no obstante, se nota una disposición a recibir más y nueva información sobre el tema.

En consecuencia, los Sistemas Contables no incluyen ningún tipo de herramienta que permita identificar los costos ambientales, y en consecuencia no se imputan al costo del producto, ni se utiliza ninguna otra técnica, sin embargo, esto no es una sorpresa, ya que el ámbito mundial los costos ambientales se encuentran en fase experimental y aún no están bien definidas las metodologías.

Los Costos Ambientales consisten en erogaciones dinerarias derivadas de la gestión ambiental empresarial, y de acuerdo a lo planteado en este artículo, debe dárseles el mismo tratamiento contable de cualquier otro costo de producción, es decir, deben imputarse al costo del producto, actividad o período. No se considera apropiado separarlos contablemente y crear un centro de costos ambientales; ya que la empresa es un ente que persigue un beneficio económico, que se genera a partir de la utilidad o ganancia del producto, y al no imputar los costos ambientales al producto generaría una base errónea para el cálculo de los beneficios. En consecuencia, el tratamiento que se sugiere es que el costo ambiental, cualquiera sea su tipo (preventivo, control y mitigación) se incorporen al costo del producto.
En otras palabras para efectos de evaluar la gestión ambiental empresarial es necesario identificar y medir por separado los costos ambientales, pero para efectos de determinar el costo del producto se les debe dar un tratamiento como cualquier otro costo vinculado al proceso productivo.

A continuación se sugieren algunos pasos a seguir en función de conformar los Costos Ambientales de la empresa:

a. Medir el impacto ambiental que se genera de la actividad productiva, identificando cada efluente o contamínate.

b. Identificar las diversas actividades de gestión ambiental destinadas a atender el impacto ambiental.

c. Clasificar las actividades de gestión ambiental, sugerimos comenzar con una clasificación de fácil de manejo, en la presente investigación se utilizó la siguiente: costos de prevención, costos de control y costos de mitigación.

d. Asociar cada una de estas actividades con los diversos procesos y departamentos de la empresa.

e. Cuantificar los recursos empleados en cada tipo de actividad y asociarlo al proceso o departamento, al igual que cualquier otro costo.

f. Llevar al costo del producto junto a los demás costos de producción.

g. Elaborar cuadros resúmenes de los costos ambientales por actividad y proceso o departamento para su análisis.

Elaborar índices para establecer la incidencia de los costos ambientales en el costo total del producto. 


\section{Referencias bibliográficas}

Ablan, Nayibe y Méndez, Elías (Enero-Junio 2004). Contabilidad y ambiente. Una disciplina para el conocimiento. Actualidad Contable. FACES-ULA. Mérida. Venezuela.

Ariza, Danilo (octubre- diciembre 2000). Una perspectiva para captar la inserción contable en la problemática medio ambiental. Revista Legis del Contador. Legis Lec editores. Bogotá. Colombia.

Bautista, Santiago (06/06/2001). Inversión de $\$ 800$ millones para siembra de palma aceitera en Zulia. Maracaibo. EI Universal. hppt/www.eluniversal.com

Bravo, M. (1997). Contabilidad y el problema medioambiental. Disponible: hppt:// orbita.starmedia.com/mjbravo/congreso.htm.

Epsteín, Marc (2000). El desempeño ambiental de la empresa. ECOE Ediciones. Santa Fe de Bogotá, Colombia.

Gray Rob, Bebbinton Jan, Walters Diana (1999). Contabilidad y auditoría ambiental. ECOE Ediciones. Santa Fe de Bogotá, Colombia.

Fundación Forum Ambiental (1999). Contabilidad ambiental: medida, evaluación y comunicación de la actuación ambiental de la empresa. Disponible en www.forumambiental.org/pdf/contab/ pdf
Marín, Carolina (s/f). La Contabilidad ambiental. Disponible en www.monografias. com/trabajos16/contabilidadambiental/contabilidadambiental $/ 5 \mathrm{htm}$

Moreno, Belkys (2001, Mayo). La palma aceitera: El otro petróleo de Venezuela. UNE-Avances (1), 4.

Rodríguez, Hugo y Yardin, Amaro (1999). ¿De qué hablamos cuando hablamos de costos medioambientales?. Ponencia presentada XXII Congreso Argentino de profesores universitarios de costos. Disponible en http://www.aurco.org.uy/biblioteca.htm.

Rodríguez, Miguel y Ricart, Joan (1998). Dirección medioambiental de la empresa. Gestión 2000.

Scavone Graciela; Ferrucci, Graciela y Schapira, Adriana (1999) Relevancia de los costos ambientales en la gestión empresarial. Disponible en hppt/www. economicas-online.com

Seoánez, Mariano (1999). Manual de gestión medioambiental de la empresa. Ediciones Mundi-Prensa. Madrid, España.

Spallarossa, L.M. (2000). Necesidad de una contabilidad ambiental. En Universo Económico, V. 10, No. 55, p. 62. Disponible en www.cpcecf.org.ar/Publicaciones/ue/ue55/15.htm

World Resources Institute (1996). La guía global del medio ambiente. Ecoespaña. Madrid. 